\title{
Corrigendum to "Association of Nuclear Factor-Erythroid 2-Related Factor 2, Thioredoxin Interacting Protein, and Heme Oxygenase-1 Gene Polymorphisms with Diabetes and Obesity in Mexican Patients"
}

\author{
Angélica Saraí Jiménez-Osorio, ${ }^{1}$ Susana González-Reyes, ${ }^{1}$ \\ Wylly Ramsés García-Niño, ${ }^{1}$ Hortensia Moreno-Macías, ${ }^{2}$ \\ Martha Eunice Rodríguez-Arellano, ${ }^{3}$ Gilberto Vargas-Alarcón, ${ }^{4}$ \\ Joaquín Zúñiga, ${ }^{5}$ Rodrigo Barquera, ${ }^{6}$ José Pedraza-Chaverri, ${ }^{1}$ \\ Juan Pablo Meza-Espinoza, ${ }^{7}$ Evelia Leal-Ugarte, ${ }^{7}$ and Valeria Peralta-Leal ${ }^{7}$ \\ ${ }^{1}$ Department of Biology, National Autonomous University of Mexico (UNAM), 04510 Mexico City, DF, Mexico \\ ${ }^{2}$ Economy Department, Autonomous Metropolitan University-Iztapalapa, 09340 Mexico City, DF, Mexico \\ ${ }^{3}$ Research Department, Regional Hospital "Lic. Adolfo López Mateos", ISSSTE, 01030 Mexico City, DF, Mexico \\ ${ }^{4}$ Molecular Biology Department, National Institute of Cardiology "Ignacio Chávez", 14080 Mexico City, DF, Mexico \\ ${ }^{5}$ Department of Immunology, National Institute of Respiratory Diseases "Ismael Cosío Villegas", 14080 Mexico City, DF, Mexico \\ ${ }^{6}$ Molecular Genetics Laboratory, National School of Anthropology and History, 14030 Mexico City, DF, Mexico \\ ${ }^{7}$ Departamento de Genética, Facultad de Medicina e Ingeniería en Sistemas Computacionales de Matamoros, \\ Universidad Autónoma de Tamaulipas, Sendero Nacional km 3, 87349 Matamoros, TAMPS, Mexico \\ Correspondence should be addressed to José Pedraza-Chaverri; pedraza@unam.mx
}

Received 20 February 2017; Accepted 28 February 2017; Published 30 May 2017

Copyright (C) 2017 Angélica Saraí Jiménez-Osorio et al. This is an open access article distributed under the Creative Commons Attribution License, which permits unrestricted use, distribution, and reproduction in any medium, provided the original work is properly cited.

In the article titled "Association of Nuclear Factor-Erythroid 2-Related Factor 2, Thioredoxin Interacting Protein, and Heme Oxygenase-1 Gene Polymorphisms with Diabetes and Obesity in Mexican Patients," [1] typing errors were found after subsequent analyses of the entire dataset. These issues were raised to the attention of the original authors by Drs. Meza-Espinoza, Leal-Ugarte, and Peralta-Leal of the Universidad Autónoma de Tamaulipas. The allelic frequencies were added and the corrected data are shown in italics in Tables 2, 3 , and 4 .

It is important to remark that the model (recessive for allele T, Table 4) was calculated taking in count that $\mathrm{CC}+$ $\mathrm{CT}=0$. When $\mathrm{CC}$ is considered as the risk allele the OR increases to 2.4 (CI: $1.28-4.64, P=0.006)$ under a dominant model $(\mathrm{CC}+\mathrm{CT}=1$ versus $\mathrm{TT}=0)$. This reveals that the
TT genotype in this study shows a protective factor against obesity and the genotype CC could be considered as the risk factor for obesity, as was stated in the original article.

The last paragraph of Section 3 should be "CC carriers had higher glucose levels in comparison with $C A+A A$ carriers when genotype was compared as dominant model."

Finally, in Section 4 (Discussion) when Wang et al. [7] is cited, the correct statement is the following:

"Individuals with CC genotype had lower total antioxidant capacity, glutathione levels, superoxide dismutase, catalase, and glutathione peroxidase activities as well as lower homeostasis model assessment of $\beta$-cell function index (HOMA- $\beta$ ) in comparison with individuals with the AA genotype." 
TABLE 2: Genotype and allele frequencies of the polymorphisms studied in diabetic patients and controls.

\begin{tabular}{|c|c|c|c|c|c|c|}
\hline Gene/polymorphism & $\begin{array}{c}\text { Genotypes } \\
\text { Alleles }\end{array}$ & $\begin{array}{c}\text { Diabetes } \\
n(\%)\end{array}$ & $\begin{array}{c}\text { Controls } \\
n(\%)\end{array}$ & OR $(95 \% \mathrm{CI})$ & $P$ & $P$ of $\mathrm{HWE}$ \\
\hline \multirow{5}{*}{$\begin{array}{l}\text { TXNIP } \\
\text { rs7211 }\end{array}$} & $\mathrm{CC}$ & $345(55.4)$ & $528(54.5)$ & Reference & & \multirow{5}{*}{0.880} \\
\hline & CT & $239(38.4)$ & $376(38.8)$ & $\begin{array}{c}0.97 \\
(0.78-1.2)\end{array}$ & 0.798 & \\
\hline & TT & $39(6.2)$ & $65(6.7)$ & $\begin{array}{c}0.92 \\
(0.60-1.39)\end{array}$ & 0.690 & \\
\hline & C & $939(74.6)$ & $1432(73.9)$ & Reference & & \\
\hline & $T$ & 317 (25.4) & $506(26.1)$ & $\begin{array}{c}0.966 \\
(1.82-1.37) \\
\end{array}$ & 0.674 & \\
\hline \multirow{5}{*}{$\begin{array}{l}\text { NQO1 } \\
\text { rs1800566 }\end{array}$} & CC & $216(34.7)$ & $327(33)$ & Reference & & \multirow{5}{*}{0.406} \\
\hline & CT & $288(46.2)$ & $483(48.6)$ & $0.90(0.7-1.1)$ & 0.373 & \\
\hline & $\mathrm{TT}$ & 119 (19.1) & $183(18.4)$ & $0.98(0.7-1.3)$ & 0.915 & \\
\hline & $C$ & $720(57.8)$ & $1137(57.25)$ & Reference & & \\
\hline & $T$ & $526(42.2)$ & $849(42.75)$ & $\begin{array}{c}0.98 \\
(0.85-1.13) \\
\end{array}$ & 0.765 & \\
\hline \multirow{5}{*}{$\begin{array}{l}\text { HMOX-1 } \\
\text { rs2071749 }\end{array}$} & AA & $269(43.8)$ & $413(43.2)$ & Reference & & \multirow{5}{*}{0.625} \\
\hline & $\mathrm{AG}$ & 267 (43.5) & $417(43.6)$ & $\begin{array}{c}0.98 \\
(0.79-1.2)\end{array}$ & 0.877 & \\
\hline & GG & $78(12.7)$ & $126(13.2)$ & $\begin{array}{c}0.95 \\
(0.69-1.3)\end{array}$ & 0.757 & \\
\hline & $A$ & 805 (65.5) & $1243(65)$ & Reference & & \\
\hline & $G$ & $423(34.5)$ & $669(35)$ & $\begin{array}{c}0.97 \\
(0.84-1.13) \\
\end{array}$ & 0.755 & \\
\hline \multirow{5}{*}{$\begin{array}{l}\text { NRF2 } \\
\text { rs2364723 }\end{array}$} & $\mathrm{CC}$ & $210(33.6)$ & $301(30.3)$ & Reference & & \multirow{5}{*}{0.092} \\
\hline & CG & $286(45.7)$ & $471(47.5)$ & $0.87(0.7-1.1)$ & 0.236 & \\
\hline & GG & $129(20.6)$ & $220(22.2)$ & $\begin{array}{c}0.84 \\
(0.63-1.1)\end{array}$ & 0.223 & \\
\hline & C & $706(56.5)$ & $1073(54)$ & Reference & & \\
\hline & G & $544(43.5)$ & $911(46)$ & $\begin{array}{c}0.91 \\
(0.79-1.05) \\
\end{array}$ & 0.182 & \\
\hline \multirow{5}{*}{$\begin{array}{l}\text { NRF2 } \\
\text { rs6721961 }\end{array}$} & $\mathrm{CC}$ & 407 (65.3) & $618(62.5)$ & Reference & & \multirow{5}{*}{0.281} \\
\hline & CA & $189(30.4)$ & $317(32)$ & $0.90(0.7-1.1)$ & 0.374 & \\
\hline & AA & $27(4.3)$ & $54(5.5)$ & $0.76(0.5-1.2)$ & 0.259 & \\
\hline & $C$ & $1003(80.5)$ & $1553(78.5)$ & Reference & & \\
\hline & $A$ & 243 (19.5) & $425(21.5)$ & $\begin{array}{c}0.88 \\
(0.74-1.05) \\
\end{array}$ & 0.176 & \\
\hline
\end{tabular}

CI, confidence interval; HWE, Hardy-Weinberg equilibrium; HMOX-1, heme oxygenase 1; NQO1, NAD(P)H quinone oxidoreductase 1; NRF2, nuclear factorerythroid 2- (NF-E2-) related factor 2; OR, odds ratio; and TXNIP, thioredoxin-interacting protein. 
TABLE 3: Genotype and allele frequencies of the polymorphisms studied in obese and nonobese subjects.

\begin{tabular}{|c|c|c|c|c|c|}
\hline Gene/polymorphism & $\begin{array}{c}\text { Genotype } \\
\text { Alleles }\end{array}$ & $\begin{array}{c}\text { Obesity } \\
n(\%)\end{array}$ & $\begin{array}{c}\text { No obesity } \\
n(\%)\end{array}$ & OR (95\% CI) & $P$ \\
\hline \multirow{5}{*}{$\begin{array}{l}\text { TRXNIP } \\
\text { rs7211 }\end{array}$} & $\mathrm{CC}$ & $350(56.6)$ & $523(53.7)$ & Reference & \\
\hline & $\mathrm{CT}$ & $239(38.7)$ & $376(38.6)$ & $\begin{array}{c}0.95 \\
(0.77-1.17)\end{array}$ & 0.633 \\
\hline & $\mathrm{TT}$ & $29(4.7)$ & $75(7.7)$ & $\begin{array}{c}0.57 \\
(0.37-0.9)\end{array}$ & 0.017 \\
\hline & C & $239(76)$ & $1422(73)$ & Reference & \\
\hline & $T$ & $297(24)$ & $526(27)$ & $0.85(0.72-1)$ & 0.061 \\
\hline \multirow{5}{*}{$\begin{array}{l}\text { NQO1 } \\
\text { rs1800566 }\end{array}$} & $\mathrm{CC}$ & $212(34.2)$ & $331(33.3)$ & Reference & \\
\hline & CT & $302(48.8)$ & 469 (47) & $1(0.8-1.25)$ & 0.963 \\
\hline & $\mathrm{TT}$ & $106(17)$ & $196(19.7)$ & $0.84(0.6-1.1)$ & 0.257 \\
\hline & $C$ & $726(58.5)$ & $1131(56.8)$ & Reference & \\
\hline & $T$ & $514(41.5)$ & $561(43.2)$ & $\begin{array}{c}0.93 \\
(0.8-1.07) \\
\end{array}$ & 0.322 \\
\hline \multirow{5}{*}{$\begin{array}{l}\text { HMOX-1 } \\
\text { rs2071749 }\end{array}$} & AA & $261(40)$ & $419(45.7)$ & Reference & \\
\hline & AG & $305(46.9)$ & $378(41.3)$ & $1.3(1-1.6)$ & 0.019 \\
\hline & GG & $85(13.1)$ & $119(13)$ & $1.1(0.8-1.5)$ & 0.399 \\
\hline & $G$ & $827(63.5)$ & $1216(66.4)$ & Reference & \\
\hline & $A$ & $475(36.5)$ & $616(36.6)$ & $\begin{array}{c}1.13 \\
(0.97-1.31) \\
\end{array}$ & 0.097 \\
\hline \multirow{5}{*}{$\begin{array}{l}\text { NRF2 } \\
\text { rs2364723 }\end{array}$} & $\mathrm{CC}$ & $194(31)$ & $317(32)$ & Reference & \\
\hline & CG & $300(48)$ & $457(46)$ & $1.1(0.85-1.35)$ & 0.551 \\
\hline & GG & $131(21)$ & $218(22)$ & $0.98(0.7-1.3)$ & 0.899 \\
\hline & $C$ & $688(55)$ & $1091(55)$ & Reference & \\
\hline & $G$ & $562(45)$ & $893(45)$ & $\begin{array}{c}0.99 \\
(0.86-1.15) \\
\end{array}$ & 0.699 \\
\hline \multirow{5}{*}{$\begin{array}{l}\text { NRF2 } \\
\text { rs6721961 }\end{array}$} & $\mathrm{CC}$ & $390(63.1)$ & $635(63.9)$ & Reference & \\
\hline & $\mathrm{CA}$ & $195(31.6)$ & $311(31.3)$ & $1(0.8-1.3)$ & 0.853 \\
\hline & AA & $33(5.3)$ & $48(4.8)$ & $1.1(0.7-1.7)$ & 0.631 \\
\hline & $C$ & 975 (78.9) & $1581(79.5)$ & Reference & \\
\hline & $A$ & $261(21.1)$ & 407 (20.5) & $\begin{array}{c}1.04 \\
(0.87-1.23)\end{array}$ & 0.661 \\
\hline
\end{tabular}

TXNIP, thioredoxin-interacting protein; NQO1, NAD(P)H quinone oxidoreductase 1; HMOX-1, heme oxygenase 1; NRF2, nuclear factor-erythroid 2- (NFE2-) related factor 2.

TABLE 4: Genotype frequency of the rs7211 polymorphism in subjects without diabetes and women.

\begin{tabular}{|c|c|c|c|c|c|c|}
\hline & Obese & Nonobese & Crude OR (95\% CI) & $P$ & Adjusted $^{\mathrm{a}}$ OR (95\% CI) & $P$ \\
\hline \multicolumn{7}{|l|}{ Nondiabetic } \\
\hline $\mathrm{CC}$ & $189(56.6)$ & $339(53.4)$ & Reference & & Reference & \\
\hline CT & $133(39.8)$ & $243(38.3)$ & $0.98(0.75-1.3)$ & 0.896 & $1(0.77-1.4)$ & 0.863 \\
\hline TT & $12(3.6)$ & $53(8.3)$ & $0.4(0.21-0.77)$ & 0.007 & $0.3(0.15-0.7)$ & 0.003 \\
\hline $\mathrm{CC}+\mathrm{CT}=0$ versus $\mathrm{TT}=1$ & $322(96.4)$ & $582(91.5)$ & $0.4(0.21-0.76)$ & 0.006 & $0.39(0.18-0.8)$ & 0.014 \\
\hline \multicolumn{7}{|l|}{ Women } \\
\hline $\mathrm{CC}$ & $197(59)$ & $259(51)$ & Reference & & Reference & \\
\hline CT & $118(36)$ & $203(40)$ & $0.7(0.6-1)$ & 0.072 & $0.9(0.6-1.2)$ & 0.418 \\
\hline TT & $17(5)$ & $47(9)$ & $0.5(0.26-0.85)$ & 0.013 & $0.5(0.25-0.96)$ & 0.04 \\
\hline $\mathrm{CT}+\mathrm{TT}$ & $135(41)$ & $250(49)$ & $0.70(0.5-0.9)$ & 0.016 & $0.7(0.5-0.96)$ & 0.028 \\
\hline
\end{tabular}

CI, confidence interval; OR, odds ratio.

${ }^{a}$ Obesity in logistic regression was adjusted by age, gender (except in women model), glucose, triglycerides, LDL-C, and HDL-C levels. 


\section{References}

[1] A. S. Jiménez-Osorio, S. González-Reyes, W. R. García-Niño et al., "Association of nuclear factor-erythroid 2-related factor 2 , thioredoxin interacting protein, and heme oxygenase-1 gene polymorphisms with diabetes and obesity in Mexican patients," Oxidative Medicine and Cellular Longevity, vol. 2016, Article ID 7367641, 8 pages, 2016. 


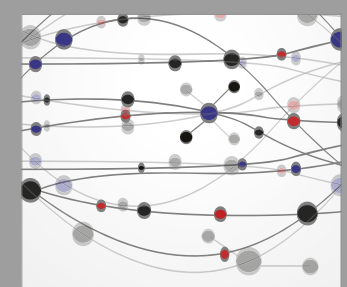

The Scientific World Journal
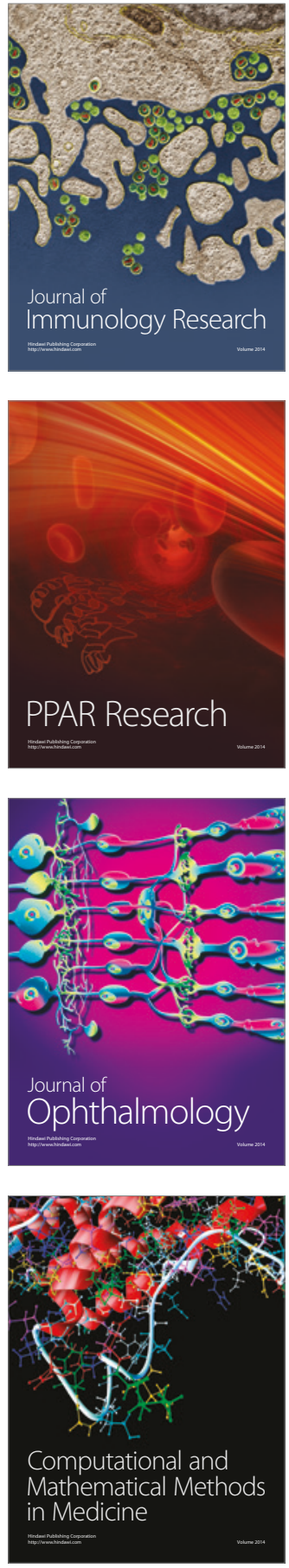

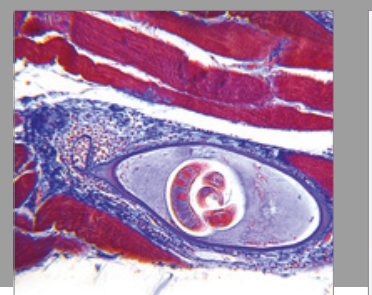

Gastroenterology Research and Practice
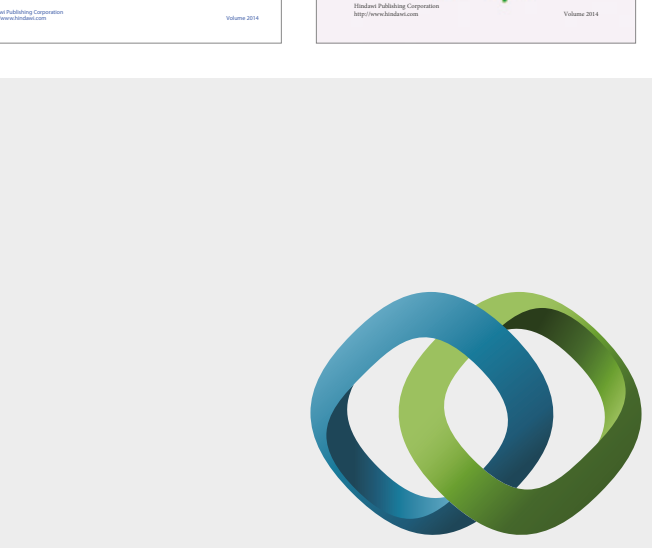

\section{Hindawi}

Submit your manuscripts at

https://www.hindawi.com
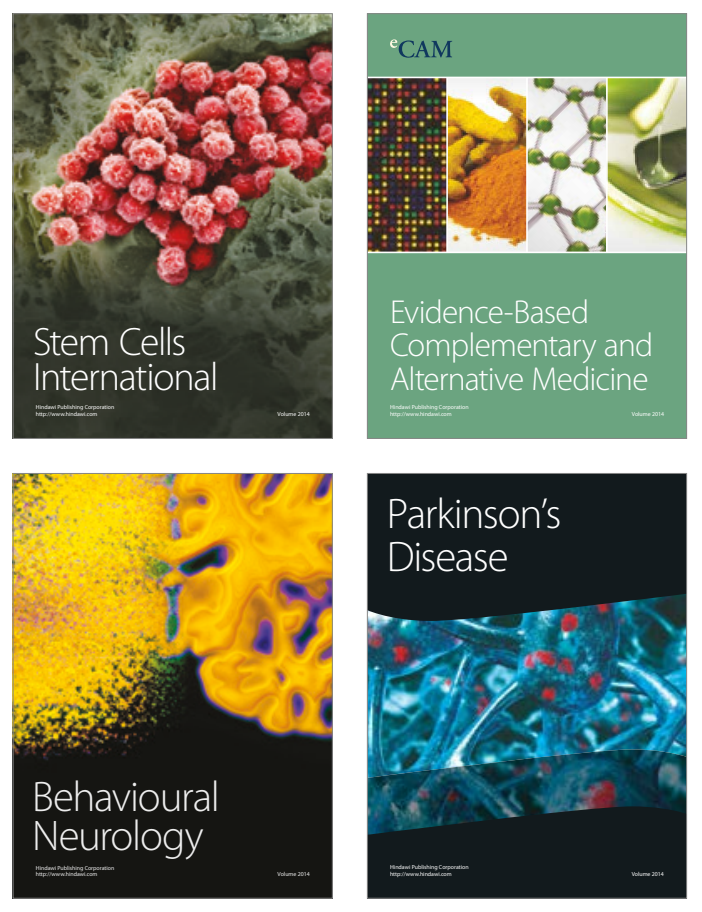
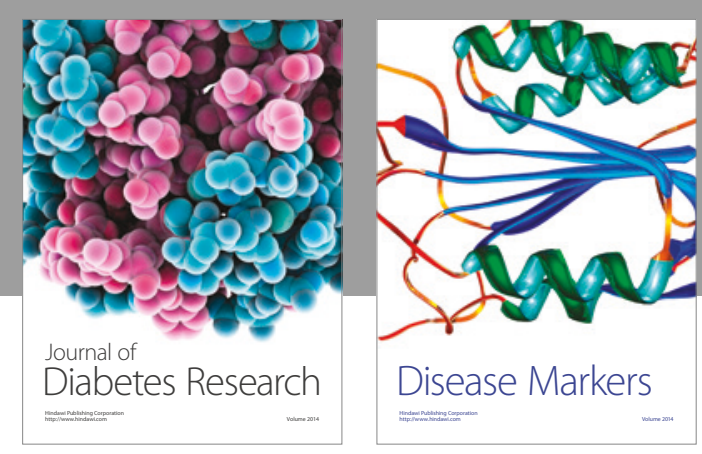

Disease Markers
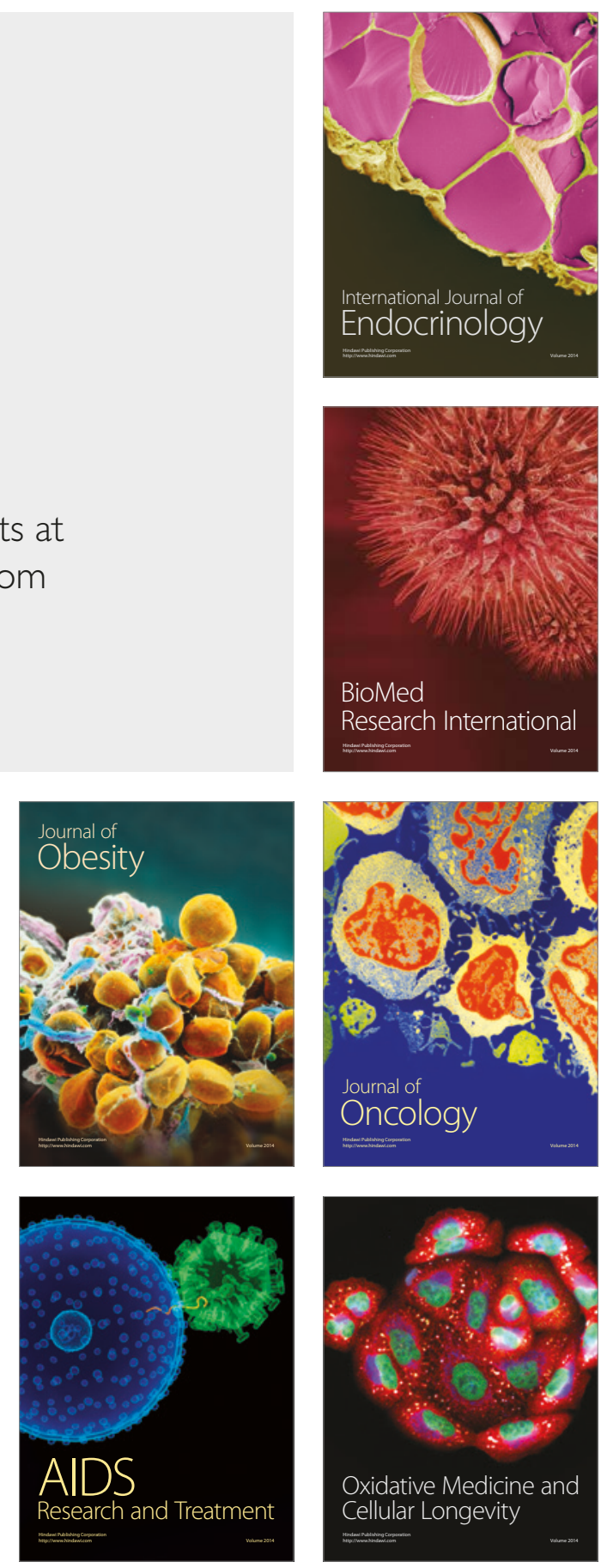